\title{
Validation and Potential of Albumin-Bilirubin Grade and Prognostication in a Nationwide Survey of 46,681 Hepatocellular Carcinoma Patients in Japan: The Need for a More Detailed Evaluation of Hepatic Function
}

\author{
Atsushi Hiraoka ${ }^{a}$ Kojiro Michitaka ${ }^{a}$ Takashi Kumada $^{b}$ Namiki Izumi ${ }^{c}$ \\ Masumi Kadoyad Norihiro Kokudo ${ }^{\text {e }}$ Shoji Kubo $^{f}$ Yutaka Matsuyamag $^{9}$ \\ Osamu Nakashima $^{h} \quad$ Michiie Sakamoto $^{i}$ Tadatoshi Takayama $^{j}$ \\ Takashi Kokudok Kosuke Kashiwabaral Masatoshi Kudom $^{m}$ \\ The Liver Cancer Study Group of Japan \\ ${ }^{a}$ Gastroenterology Center, Ehime Prefectural Central Hospital, Matsuyama, b Department \\ of Gastroenterology, Ogaki Municipal Hospital, Ogaki, ' Department of Gastroenterology, \\ Musashino Red Cross Hospital, Tokyo, dDepartment of Radiology, Shinshu University \\ School of Medicine, Matsumoto, eNational Center for Global Health and Medicine, Tokyo, \\ ${ }^{f}$ Department of Hepato-Biliary-Pancreatic Surgery, Osaka City University Graduate School \\ of Medicine, Osaka, ${ }^{9}$ Department of Biostatistics, School of Public Health, University of \\ Tokyo, Tokyo, h Department of Clinical Laboratory Medicine, Kurume University Hospital, \\ Kurume, i Department of Pathology, Keio University School of Medicine, jDepartment of \\ Digestive Surgery, Nihon University School of Medicine, and ${ }^{k}$ Hepato-Biliary-Pancreatic \\ Surgery Division, Department of Surgery, Graduate School of Medicine, and 'Department \\ of Biostatistics, School of Public Health, Graduate School of Medicine, University of Tokyo, \\ Tokyo, and mepartment of Gastroenterology and Hepatology, Kindai University School of \\ Medicine, Osaka, Japan
}

\section{Keywords}

Hepatocellular carcinoma - Albumin-bilirubin grade - TNM stage - Japan Integrated Staging · Prognosis · Modified albumin-bilirubin grade

\footnotetext{
Abstract

Background/Aim: Recently, albumin-bilirubin (ALBI) scoring/grading, consisting of only albumin and total bilirubin, has been proposed. We examined the efficacy of this grading system for determining hepatic function in patients with hepatocellular carcinoma (HCC). Methods/Materials: The prognoses of 46,681 HCC patients based on results obtained from a nationwide survey conducted in Japan from 2001 to 2007 were evaluated using (1) Japan 
Integrated Staging (JIS), consisting of Child-Pugh classification and TNM staging (TNM), (2) modified JIS (m-JIS), consisting of liver damage grading and TNM, and (3) ALBI-TNM (ALBI-T), consisting of ALBI grading and TNM, and the results were compared. A subanalysis was also performed to define a cutoff value for ALBI scores for a more detailed stratification of hepatic function. Results: ALBI-T, JIS, and m-JIS each showed good capacity for the stratification of prognoses. Although the Akaike information criterion for ALBI-T was nearly equal to that for JIS and m-JIS, the Kaplan-Meier curves and median survival times obtained with ALBI-T were always superior to the corresponding scores. When the indocyanine green retention test $(<30 \%)$ was used as an additional cutoff value for ALBI score $(-2.270$, area under the curve 0.828 ) to divide ALBI grade into 4 levels (modified ALBI [mALBI] grade), mALBI grade was able to stratify the prognosis of patients at any TNM stage in order of grade. Modified ALBI-T (mALBI-T), using mALBI grading and TNM, produced a more detailed stratification for prognosis. Conclusion: The predictive value for prognosis of ALBI-T was found to be equal to that of JIS and m-JIS. In addition, mALBI grading and mALBI-T, as proposed in the present study, might provide a more detailed assessment of the hepatic function and prognosis of HCC patients.

(C) 2017 S. Karger AG, Basel

\section{Introduction}

The Child-Pugh classification [1] is used worldwide as a standard assessment tool for hepatic function in hepatocellular carcinoma (HCC) patients, while it is also a part of the Evidence-Based Clinical Practice Guidelines for HCC of the Japan Society of Hepatology (JSH) as well as liver damage grading [2,3]. Recently, a new assessment tool for hepatic function, albumin-bilirubin (ALBI) grading, which consists of only albumin and total bilirubin, has been proposed [4]. As compared to other assessment methods, ALBI grade, simply based on 2 common serological factors and determined by serial ALBI scoring, is thought to be advantageous, because the score can also be arbitrarily divided into more detailed grades when necessary.

As a total staging system for HCC, the Japan Integrated staging (JIS) system has also been proposed [5], which consists of the Child-Pugh classification and TNM staging of the Liver Cancer Study Group of Japan (LCSGJ) [6, 7]; JIS has been shown to have a better ability to predict prognosis than the Cancer of the Liver Italian Program (CLIP) scoring system [8, 9]. On the other hand, the usefulness of modified JIS (m-JIS) for grading liver damage as compared to JIS has been proposed for patients who undergo an indocyanine green retention test (ICGr15) [10]. However, some issues regarding the analysis of hepatic function with the ICG-r15 have been reported, including problems related to the requirement of injection, the risk of anaphylactic shock, and difficulties with obtaining accurate results for patients with marked constitutional jaundice or a portosystemic shunt [11]. As a result, data for the ICG-r15 obtained from a retrospective cohort are often lacking. Recently, ALBI-TNM scoring (ALBI-T), which is composed of JIS used with ALBI grading, was reported to have good prognostic value in 2,584 Japanese HCC patients treated at 2 different institutions [12].

To evaluate whether ALBI grading can be used for hepatic functional assessment instead of Child-Pugh classification and liver damage grading, and to establish a more useful method for the assessment of hepatic function, we compared ALBI grading, Child-Pugh classification, and liver damage grading in the present study. In addition, we examined ALBI scoring/ grading for its ability to provide a more detailed evaluation of hepatic function and predict prognosis in Japanese HCC patients using data from a nationwide survey. 
Hiraoka et al.: Validation and Potential of ALBI Grade and Prognostication in a Nationwide Survey of 46,681 HCC Patients in Japan

\section{Subjects and Methods}

\section{Patients}

From January 2001 to December 2007, details regarding 64,928 treatment-naïve patients with HCC in Japan were recorded using a nationwide survey system. Of these patients, we analyzed 46,681 in the present study, after exclusion of cases lacking serological (albumin, total bilirubin, and prothrombin time) or clinical data (information about ascites or hepatic coma) for Child-Pugh classification or LCSGJ TNM staging. In patients positive for anti-hepatitis $\mathrm{C}$ virus (HCV) or hepatitis B surface antigen, $\mathrm{HCC}$ was considered to be due to HCV or hepatitis B virus (HBV), respectively. Those negative for both anti-HCV and hepatitis B surface antigen were judged as having nonviral (nonBnonC) HCC.

\section{Assessment Methods for Hepatic Function and Prognosis}

Child-Pugh classification, liver damage grading, and ALBI grading were used for assessment of hepatic function. ALBI score and ALBI grade were defined as follows: $\left(\log _{10}\right.$ bilirubin $\left.[\mu \mathrm{mol} / \mathrm{L}] \times 0.66\right)+($ albumin [g/L] $\times-0.085$ ) (grade 1, 2, and $3=\leq-2.60,>-2.60$ to -1.39 , and $>-1.39$, respectively) [4]. The prognoses of the 46,681 patients with HCC (total group) were assessed using ALBI-T and JIS, while those of the 31,011 patients after excluding those without ICG-r15 results (ICG group) were evaluated using ALBI-T, JIS, and $\mathrm{m}$-JIS. In addition, we compared the potential for predicting prognosis of each score (ALBI-T, JIS, and m-JIS) in HCC patients within the Milan criteria (single lesion $\leq 5 \mathrm{~cm}$, or 2 or 3 lesions each $3 \mathrm{~cm}$ in size) [13] who were treated with curative therapy (surgical resection, ablative treatment) in both groups. Percutaneous ethanol injection $[14,15]$ and mainly radiofrequency ablation [16-18] were performed as ablative therapy methods.

As an additional analytic technique, we evaluated the cutoff value for ALBI score by dividing ALBI grade 2 into subgrades ( $2 \mathrm{a}$ and $2 \mathrm{~b}$ ) obtained with the ICG-r15 to produce a modified ALBI grade (mALBI grade) divided into 4 subgrades, because ALBI score is serial and ALBI grade 2 is thought to have a wide range. To evaluate mALBI grading for its ability to assess hepatic function, the prognostic predictive value of modified ALBI-T (mALBI-T), calculated using mALBI grade and LCSG) TNM stage (mALBI grade 1, 2a, 2b, and $3=$ score $0,1,2$, and 3, respectively; LCSGJ TNM stage I, II, III, and IV = score $0,1,2$, and 3, respectively), was examined in both groups.

\section{Statistical Analysis}

Prognosis was analyzed using a log-rank test with the Kaplan-Meier method. The discriminatory abilities of the scoring models were assessed using the Akaike information criterion (AIC) [19]. Pearson test, receiver operating characteristic, and area under the curve (AUC) values were calculated for comparisons between ALBI score and the ICG-r15. For multiple comparisons, Holm's method was used. All statistical analyses were performed using Easy R (EZR) version 1.29 (Saitama Medical Center, Jichi Medical University, Saitama, Japan) [20], a graphical user interface for the R statistical Computing Environment (R Foundation, Vienna, Austria). All $p$ values were derived from two-tailed tests, with $p<0.05$ accepted as statistically significant.

\section{Results}

The clinical features of the patients in the total group $(n=46,681)$ are shown in Table 1 . The median age was 68 years (interquartile range: 61-74); 33,316 were male and 13,365 were female (HCV, HBV, HBV and HCV, nonBnonC, and unknown: $n=30,478,6,124,821$, 8,743, and 515, respectively; Child-Pugh class A, B, and C: $n=34,829,10,111$, and 1,741, respectively; LCSGJ TNM stage I, II, III, and IV: $n=7,783,21,018,12,843$, and 5,037, respectively [IVa and IVb: $n=2,927$ and 2,110, respectively]; ALBI grade 1, 2, and 3: $n=15,968$, 27,771 , and 2,942, respectively). Surgical resection was performed on 14,551 patients as the initial treatment, while it was ablative therapy for 13,359, transcatheter arterial chemoembolization [21] for 13,995, and other treatments or none for 4,776. The number of patients within the Milan criteria was 25,814 (55.3\%).

The distribution of ALBI grades for each Child-Pugh score is shown in Figure 1a. The patients classified as Child-Pugh class A consisted of 15,744 with ALBI grade 1 and 19,085 


\section{Liver Cancer}

Table 1. Clinical features of the patients ( $n=46,681$; total group)

\begin{tabular}{l|l}
\hline Liver Cancer 2017;6:325-336 \\
\hline DOI: 10.1159/000479984 & $\begin{array}{l}\text { @ 2017 S. Karger AG, Basel } \\
\text { www.karger.com/lic }\end{array}$ \\
\hline
\end{tabular}

Hiraoka et al.: Validation and Potential of ALBI Grade and Prognostication in Nationwide Survey of 46,681 HCC Patients in Japan

Median age (IQR), years

Gender

Male

Female

Etiology

HCV

HBV

HBV and HCV

nonBnonC

Unknown

Child-Pugh class

A

B

C

ALBI grade

2

3

Liver damage grade

A

B

C

No data

Median albumin (IQR), g/dL

Median total bilirubin (IQR), mg/dL

Median prothrombin time (IQR), \%

Median platelet count (IQR), $\times 10^{4} / \mu \mathrm{L}$

Median ICG-r15 rate (IQR) ${ }^{1}, \%$

Median tumor size (IQR), cm

Tumor number

\section{Single}

Multiple

No data

Vascular invasion of tumor present, $n(\%)$

Extrahepatic metastasis present, $n(\%)$

\section{LCSGJ TNM stage}

$$
\text { I }
$$

II

III

IVa

IVb

Treatment

$\begin{array}{lr}\text { Resection } & 14,551 \\ \text { Ablative therapies } & 13,359 \\ \text { TACE } & 13,995 \\ \text { Others } & 2,341 \\ \text { None } & 2,435\end{array}$

IQR, interquartile range; $\mathrm{HCV}$, hepatitis $\mathrm{C}$ virus; $\mathrm{HBV}$, hepatitis $\mathrm{B}$ virus; nonBnonC, negative for both HBV and HCV; ALBI, albumin-bilirubin; ICG-r15, indocyanine green retention test; LCSGJ, Liver Cancer Study Group of Japan; TACE, transcatheter arterial chemoembolization. ${ }^{1} n=31,011$ (ICG group). 
Hiraoka et al.: Validation and Potential of ALBI Grade and Prognostication in a Nationwide Survey of 46,681 HCC Patients in Japan

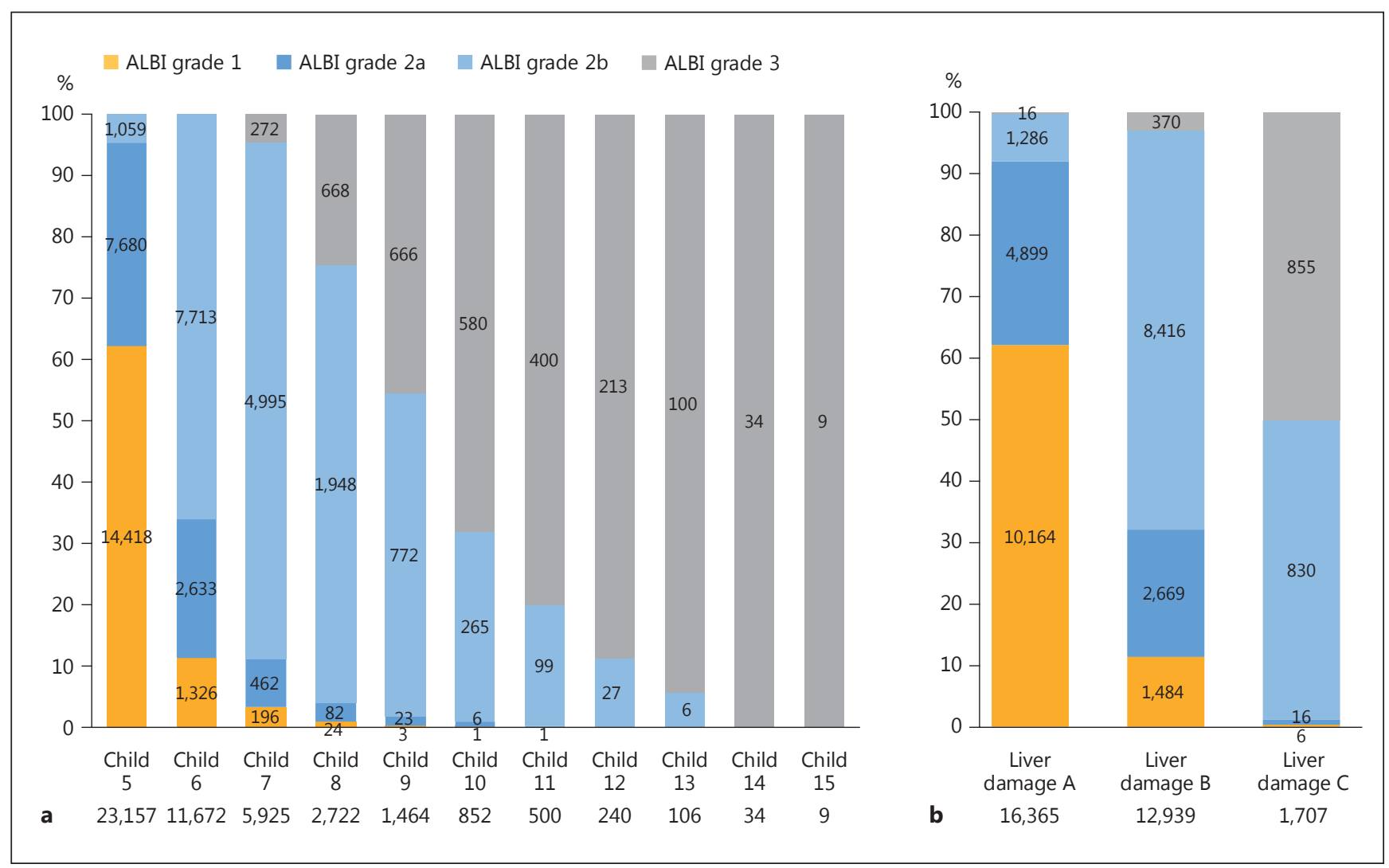

Fig. 1. a Distribution of albumin-bilirubin (ALBI) grade for each Child-Pugh score in the total group. b Distribution of ALBI grades for each liver damage grade in the ICG group.

Fig. 2. Kaplan-Meier curves for albumin-bilirubin (ALBI) grades and TNM staging scores of the Liver Cancer Study Group of Japan (ALBI-T) (solid lines) and Japan Integrated Staging (JIS) scores (broken lines) in the total group. The scores for ALBI-T were always superior to the corresponding JIS scores.

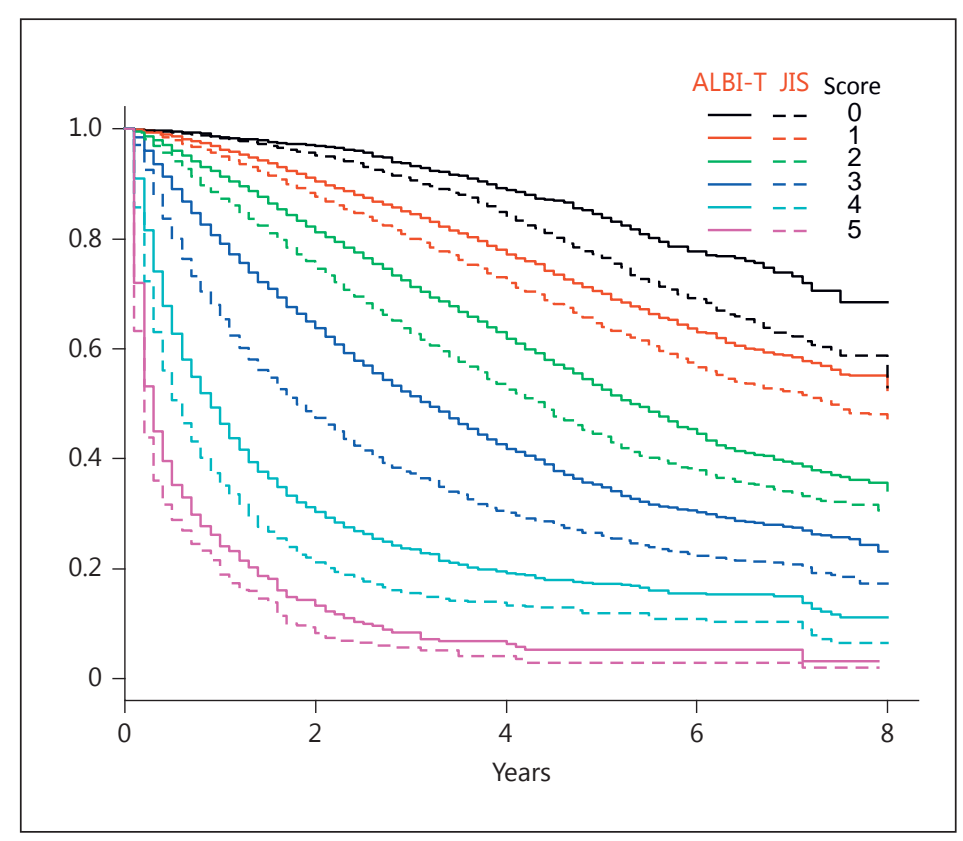


Table 2. Comparison of MST between ALBI-T, JIS, and mALBI-T among Japanese hepatocellular carcinoma patients ( $n=46,681$; total group) and those within the Milan criteria treated curatively $(n=18,886)$

\begin{tabular}{|c|c|c|c|c|c|c|c|c|c|}
\hline \multirow[t]{2}{*}{ Score } & \multicolumn{3}{|c|}{ ALBI-T } & \multicolumn{3}{|l|}{ JIS } & \multicolumn{3}{|c|}{ mALBI-T } \\
\hline & $\begin{array}{l}\text { MST, } \\
\text { years }\end{array}$ & $\begin{array}{l}95 \% \mathrm{CI} \text {, } \\
\text { years }\end{array}$ & $n$ & $\begin{array}{l}\text { MST, } \\
\text { years }\end{array}$ & $\begin{array}{l}95 \% \mathrm{CI} \text {, } \\
\text { years }\end{array}$ & $n$ & $\begin{array}{l}\text { MST, } \\
\text { years }\end{array}$ & $\begin{array}{l}95 \% \mathrm{CI}, \\
\text { years }\end{array}$ & $n$ \\
\hline \multicolumn{10}{|c|}{ All patients $(n=46,681)$} \\
\hline 0 & NA & NA-NA & 2,625 & NA & 8.0-NA & 5,908 & NA & NA-NA & 2,625 \\
\hline 1 & NA & 8.0-NA & 12,824 & 7.4 & $7.1-8.0$ & 18,242 & NA & NA-NA & 10,289 \\
\hline 2 & 5.4 & $5.2-5.6$ & 16,435 & 4.4 & $4.2-4.5$ & 13,531 & 6.0 & $5.8-5.6$ & 12,354 \\
\hline 3 & 3.2 & $3.0-3.3$ & 10,096 & 1.8 & $11.7-2.0$ & 6,430 & 4.2 & $4.0-4.4$ & 11,334 \\
\hline 4 & 0.9 & $0.9-1.0$ & 4,017 & 0.6 & $0.5-0.06$ & 2,036 & 2.5 & $2.4-2.6$ & 6,543 \\
\hline 5 & 0.3 & $0.2-0.3$ & 684 & 0.2 & $0.2-0.2$ & 534 & 0.8 & $0.7-0.9$ & 2,852 \\
\hline 6 & - & - & - & - & - & - & 0.3 & $0.2-0.3$ & 684 \\
\hline AIC & \multicolumn{3}{|c|}{$256,952.4$} & \multicolumn{3}{|c|}{$256,356.7$} & \multicolumn{3}{|c|}{$256,955.5$} \\
\hline \multicolumn{10}{|c|}{ Patients within the Milan criteria treated curatively $(n=18,886)$} \\
\hline 0 & NA & NA-NA & 2,178 & NA & NA-NA & 4,818 & NA & NA-NA & 2,178 \\
\hline 1 & NA & 8-NA & 8,465 & NA & 7.8-NA & 10,637 & NA & NA-NA & 6,568 \\
\hline 2 & 6.1 & $5.8-6.5$ & 6,958 & 5.3 & $4.9-5.8$ & 2,959 & 7.1 & 6.5-NA & 5,568 \\
\hline 3 & 4.7 & $4.4-5.6$ & 1,223 & 3.8 & $3.3-4.5$ & 453 & 5.2 & $5.0-5.7$ & 3,707 \\
\hline 4 & 3.3 & 2.2-NA & 62 & NA & $1.2-\mathrm{NA}$ & 19 & 4.2 & $3.7-4.6$ & 803 \\
\hline 5 & - & - & - & - & - & - & 3.3 & 2.2-NA & 62 \\
\hline AIC & \multicolumn{3}{|c|}{$57,365.5$} & \multicolumn{3}{|c|}{$57,416.3$} & \multicolumn{3}{|c|}{$57,133.3$} \\
\hline
\end{tabular}

ALBI-T, albumin-bilirubin grading and TNM staging; JIS, Japan Integrated Staging; mALBI-T, modified ALBI-T; MST, median survival time; NA, not available; AIC, Akaike information criterion.

with ALBI grade 2, while those classified as Child-Pugh class B were composed of 223 with ALBI grade 1, 8,282 with ALBI grade 2, and 1,606 with ALBI grade 3; the patients classified as Child-Pugh class C contained 1 with ALBI grade 1, 404 with ALBI grade 2, and 1,336 with ALBI grade 3. Even among the Child-Pugh class B patients, the rate of ALBI grade 3 was not small.

The Kaplan-Meier curves for JIS and ALBI-T of the total group $(n=46,681)$ are shown in Figure 2. The AIC value for ALBI-T for the total group was 256,952.4, while that for JIS was 256,356.7, and the Kaplan-Meier curves and median survival time (MST) for each ALBI-T score were superior to those for JIS (Fig. 2; Table 2).

The subanalysis of 31,011 of the patients in the total group with data for the ICG-r15 (ICG group) showed that the ICG-r15 had a good correlation with ALBI score ( $r=0.563 ; 95 \% \mathrm{CI}$ : $0.550-0.570 ; p<0.0001)$. Among the 31,011 patients with results for the ICG-r15, surgical resection was performed on 13,487 , while it was ablative therapy for 7,669 , transcatheter arterial chemoembolization for 7,761, and other treatments or non for 2,094. The number of patients within the Milan criteria was 17,188 (55.4\%). The cutoff value for ALBI score for the ICG-r15 (<30\%), an indicator for performance of a subsegmentectomy in the Makuuchi criteria [22, 23], was -2.270 (AUC 0.828; 95\% CI: 0.823-0.833) (Fig. 3a). The distribution of ALBI grades among the grades for liver damage is shown in Figure 1b. ALBI grade 3 was mainly included in liver damage grade $C$, while the frequency of ALBI grade 3 was very low among the patients with liver damage grade B. When ALBI grade 2 was divided into 2 subgroups (ALBI grades $2 \mathrm{a}$ and $2 \mathrm{~b})$ according to the cutoff value for the ICG-r15 $(<30 \%)$ (ALBI score: -2.270 ), thus conducting a division into 4 ALBI grades (mALBI grade), the ratio of the better subgroup (ALBI grade 2a: score <-2.270) became lower when the Child-Pugh 


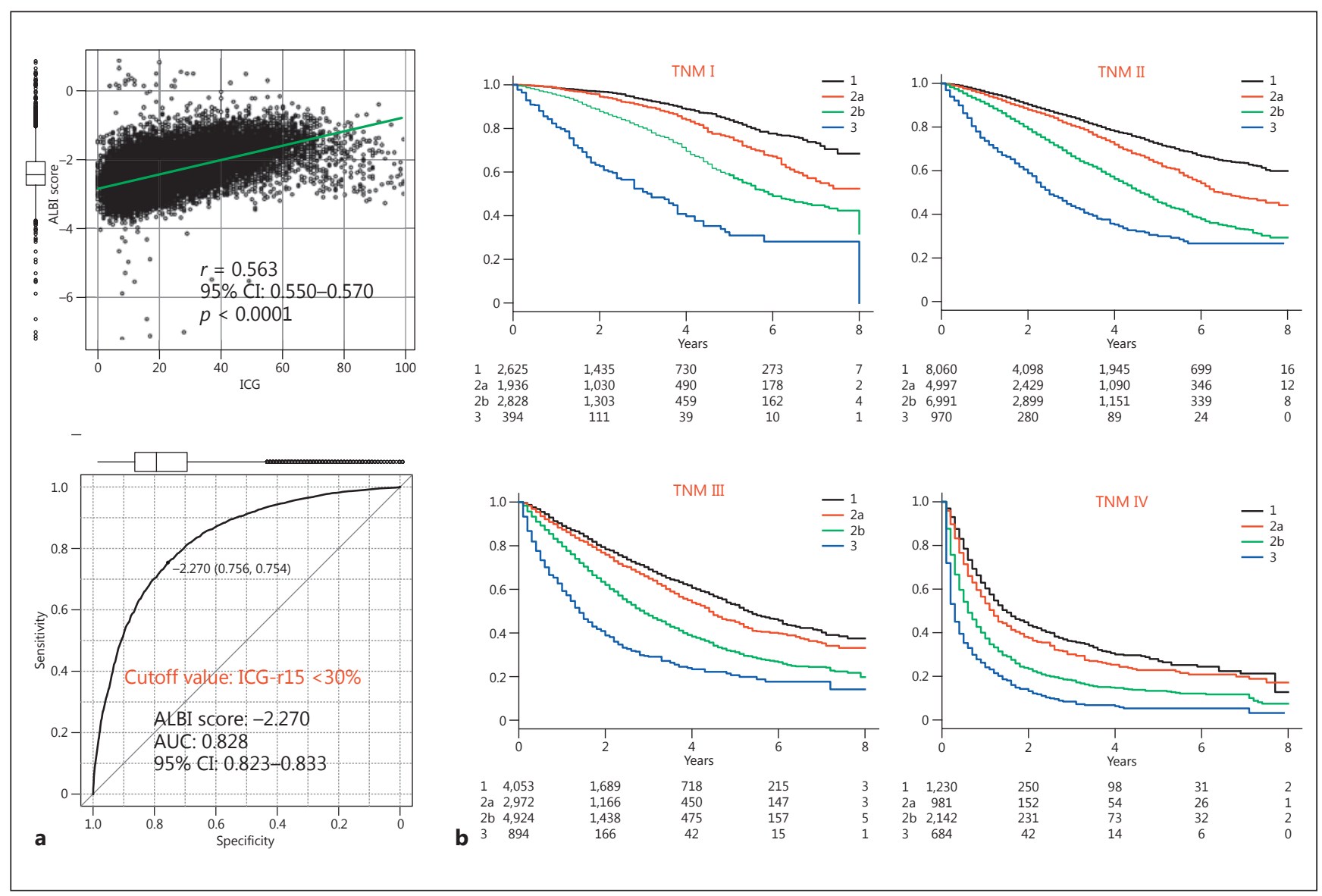

Fig. 3. a A good relationship ( $r=0.563 ; 95 \%$ CI: 0.550-0.570; $p<0.0001$ ) was observed between indocyanine green retention test (ICG-r15) and albumin-bilirubin (ALBI) score results. The cutoff value for ALBI score for the ICG-r15 (<30\%) was -2.270 (AUC 0.828; 95\% CI: 0.823-0.833). b For each Liver Cancer Study Group of Japan (LCSGJ) TNM stage, good stratification for prognosis of patients with each modified ALBI (mALBI) grade was shown. There were significant differences between each mALBI grade for each LCSGJ TNM stage, as shown by multiple comparisons with Holm's method $(p<0.01)$.

score became larger (Fig. 1a). In each TNM stage, a good ability to stratify prognosis according to the newly established subgroups for ALBI grade (mALBI grades $1,2 \mathrm{a}, 2 \mathrm{~b}$, and 3) was observed, and there was a statistically significant difference between each mALBI grade in all stages for prognosis ( $p<0.01$, Holm's method) (Fig. 3b). When an analysis was performed with mALBI grade in the same manner as with ALBI-T and JIS, patient prognosis could be stratified with the additional grades provided by mALBI-T (Fig. 4). In addition to a greater number of scores for stratification, use of mALBI-T also resulted in a better MST for each score as compared to the corresponding scores for ALBI-T and JIS in the total group (Table 2).

For the 31,011 patients in the ICG group, the AIC value for ALBI-T was similar to that for JIS and $\mathrm{m}$-JIS $(157,696.4,157,591.4$, and 157,630.9, respectively), while that for mALBI-T was 157,668.7. mALBI-T showed a better MST for each score as compared to the corresponding scores for ALBI-T, JIS, and m-JIS in the ICG group (Table 3). In the ICG group, those for ALBI-T, JIS, m-JIS, and mALBI-T for the HCC patients within the Milan criteria $(n=17,188)$ were $60,213.2,60,258.3,60,279.7$, and 59,971.2, respectively (data not shown), while those for the patients treated curatively ( $n=21,156$ ) were 83,595.4, 83,637.2, 83,611.8, and 83,500.6, respectively (data not shown), and those for the patients within the Milan criteria and treated 
Modified ALBI-T score

\begin{tabular}{|c|c|c|c|c|}
\hline Score & 0 & 1 & 2 & 3 \\
\hline mALBI grade & 1 & $2 \mathrm{a}$ & $2 \mathrm{~b}$ & 3 \\
\hline TNM stage & I & II & III & IV \\
\hline
\end{tabular}

\begin{tabular}{|c|c|c|c|c|}
\hline & $\begin{array}{c}\text { TNM } \\
\text { I }\end{array}$ & $\begin{array}{c}\text { TNM } \\
\text { II }\end{array}$ & $\begin{array}{c}\text { TNM } \\
\text { II }\end{array}$ & $\begin{array}{c}\text { TNM } \\
\text { IV }\end{array}$ \\
\hline $\begin{array}{c}\text { mALBI } \\
1\end{array}$ & 0 & 1 & 2 & 3 \\
\hline $\begin{array}{c}\text { mALBI } \\
2 \mathrm{a}\end{array}$ & 1 & 2 & 3 & 4 \\
\hline $\begin{array}{c}\text { mALBI } \\
2 \mathrm{~b}\end{array}$ & 2 & 3 & 4 & 5 \\
\hline $\begin{array}{c}\text { mALBI } \\
3\end{array}$ & 3 & 4 & 5 & 6 \\
\hline
\end{tabular}

a

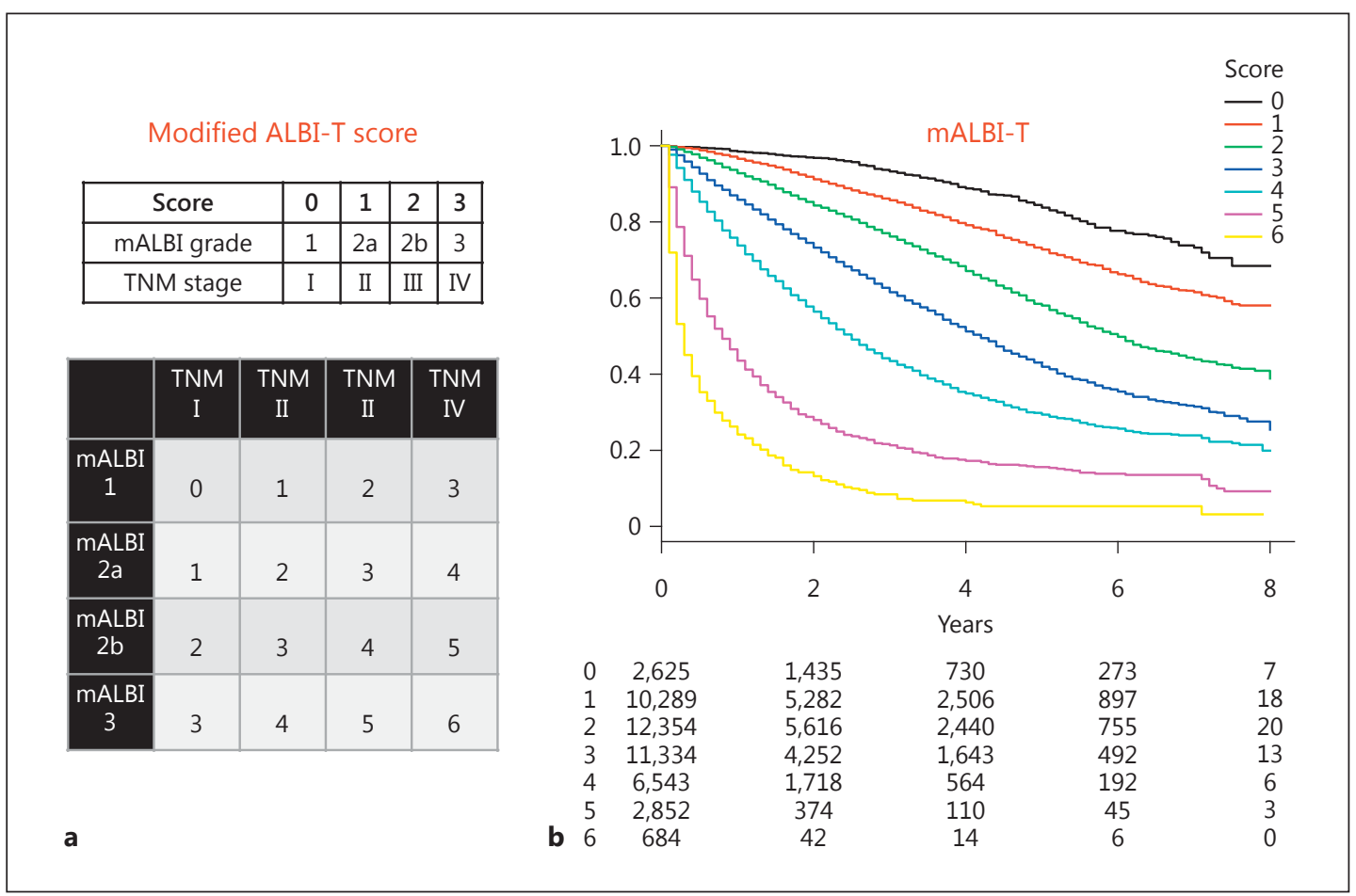

Fig. 4. a Scores for each factor (modified albumin-bilirubin [mALBI] grades $1,2 \mathrm{a}, 2 \mathrm{~b}$, and $3=\operatorname{scores} 0,1,2$, and 3; Liver Cancer Study Group of Japan TNM stages I, II, III, and IV = scores 0, 1, 2, and 3), mALBI grade, and TNM stage. Those were summed to calculate the mALBI-T score (scores 0-6). b Kaplan-Meier curves for mALBI-T showing good stratification for prognosis, as shown by each mALBI-T score in the total group $(n=$ 46,681).

curatively $(n=13,404)$ were 41,054.6, 41,107.9, 41,094.1, and 40,887.7, respectively (Table 3 ). For the 18,886 patients within the Milan criteria treated curatively in the total group, those for ALBI-T, JIS, and mALBI-T were 57,365.5, 57,416.3, and 57,133.3, respectively (Table 2).

\section{Discussion}

For treatment of HCC patients, use of a total staging system which integrates both liver function stage and tumor burden stage has become important for accurate prediction of prognosis, as it provides good information for decision-making regarding therapy. Various staging systems have been reported, such as those investigated in the Okuda [24], CLIP [8], Tokyo [25], BALAD [26], and BALAD2 [27, 28] studies. Kudo proposed JIS as a useful prognostic staging system, and showed its consistency with Child-Pugh classification and the TNM staging of the LCSGJ [5, 9]. However, there are some issues, including the fact that the Child-Pugh classification has subjective (ascites, hepatic coma) and confounding factors (albumin, ascites) and was not constructed in a statistical manner. Moreover, albumin, total bilirubin, and prothrombin time are treated as semiquantitative factors in that classification. On the other hand, ALBI-T, which consists of ALBI grading and TNM staging, has been proposed as a new total staging system for HCC [12], and validation studies have already been reported [29, 30].

In the present cohort, ALBI-T showed an almost similar AIC to that of JIS for the total group, as well as for those within the Milan criteria and treated curatively. However, the MST 
Table 3. Comparison of MST between ALBI-T, JIS, m-JIS, and mALBI-T ( $n=31,011$; ICG group) and those within the Milan criteria treated curatively $(n=13,404)$ among hepatocellular carcinoma patients in whom the ICG-r15 was examined

\begin{tabular}{|c|c|c|c|c|c|c|c|c|c|c|c|c|}
\hline \multirow[t]{2}{*}{ Score } & \multicolumn{3}{|c|}{ ALBI-T } & \multicolumn{3}{|l|}{ JIS } & \multicolumn{3}{|l|}{ m-JIS } & \multicolumn{3}{|c|}{ mALBI-T } \\
\hline & $\begin{array}{l}\text { MST, } \\
\text { years }\end{array}$ & $\begin{array}{l}95 \% \mathrm{CI} \text {, } \\
\text { years }\end{array}$ & $n$ & $\begin{array}{l}\text { MST, } \\
\text { years }\end{array}$ & $\begin{array}{l}95 \% \mathrm{CI} \text {, } \\
\text { years }\end{array}$ & $n$ & $\begin{array}{l}\text { MST, } \\
\text { years }\end{array}$ & $\begin{array}{l}95 \% \mathrm{CI} \text {, } \\
\text { years }\end{array}$ & $n$ & $\begin{array}{l}\text { MST, } \\
\text { years }\end{array}$ & $\begin{array}{l}95 \% \mathrm{CI}, \\
\text { years }\end{array}$ & $n$ \\
\hline \multicolumn{13}{|c|}{ Patients in whom the ICG-r15 was examined $(n=31,011)$} \\
\hline 0 & NA & NA-NA & 1,615 & NA & NA-NA & 3,584 & NA & NA-NA & 2,208 & NA & NA-NA & 1,615 \\
\hline 1 & NA & NA-NA & 8,962 & 8.0 & 7.4-NA & 13,206 & 7.8 & 7.3-NA & 14,387 & NA & NA-NA & 7,532 \\
\hline 2 & 5.6 & $5.4-5.8$ & 11,520 & 4.5 & $4.4-4.7$ & 9,412 & 4.8 & $4.6-5.0$ & 7,152 & 6.2 & $6.0-6.5$ & 8,685 \\
\hline 3 & 3.5 & $3.3-3.6$ & 6,677 & 2.0 & $1.9-2.2$ & 3,842 & 3.2 & $3.0-3.4$ & 5,302 & 4.4 & $4.2-4.5$ & 7,574 \\
\hline 4 & 1.1 & $1.0-1.2$ & 2,042 & 0.7 & $0.6-0.8$ & 872 & 1.1 & $1.0-1.2$ & 1,734 & 2.7 & $2.5-2.9$ & 4,073 \\
\hline 5 & 0.5 & $0.4-0.7$ & 195 & 0.4 & $0.2-0.7$ & 95 & 0.4 & $0.3-0.5$ & 228 & 1.0 & $0.9-1.1$ & 1,337 \\
\hline 6 & - & - & - & - & - & - & - & - & - & 0.5 & $0.4-0.7$ & 195 \\
\hline AIC & \multicolumn{3}{|c|}{$157,696.4$} & \multicolumn{3}{|c|}{$157,591.4$} & \multicolumn{3}{|c|}{$157,630.9$} & \multicolumn{3}{|c|}{$157,668.7$} \\
\hline \multicolumn{13}{|c|}{ Patients within the Milan criteria treated curatively in whom the ICG-r15 was examined $(n=13,404)$} \\
\hline 0 & NA & NA-NA & 1,390 & NA & NA-NA & 3,034 & NA & NA-NA & 1,916 & NA & NA-NA & 1,390 \\
\hline 1 & NA & NA-NA & 6,041 & NA & NA-NA & 8,041 & NA & NA-NA & 9,025 & NA & NA-NA & 4,946 \\
\hline 2 & 6.2 & $5.9-6.8$ & 5,118 & 5.2 & $4.9-5.8$ & 2,049 & 5.5 & $4.9-5.8$ & 1,868 & 7.3 & 6.7-NA & 3,931 \\
\hline 3 & 4.7 & $4.4-5.2$ & 819 & 3.5 & $2.8-4.2$ & 274 & 4.6 & $4.2-5.2$ & 541 & 5.2 & $4.9-5.6$ & 2,591 \\
\hline 4 & 2.6 & $1.9-4.0$ & 36 & 2.5 & $1.2-\mathrm{NA}$ & 6 & 2.5 & $1.9-4.5$ & 54 & 4.2 & $3.5-4.6$ & 510 \\
\hline 5 & - & - & - & - & - & - & - & - & - & 2.6 & $1.9-4.0$ & 36 \\
\hline AIC & \multicolumn{3}{|c|}{$41,054.6$} & \multicolumn{3}{|c|}{$41,107.9$} & \multicolumn{3}{|c|}{$41,094.1$} & \multicolumn{3}{|c|}{$40,887.7$} \\
\hline
\end{tabular}

ICG-r15, indocyanine green retention test; ALBI-T, albumin-bilirubin grading and TNM staging; JIS, Japan Integrated Staging; m-JIS, modified JIS; mALBI-T, modified ALBI-T; MST, median survival time; NA, not available; AIC, Akaike information criterion.

for each ALBI-T score was superior to that for the corresponding JIS score. Although ALBI grade is calculated with only those 2 factors, ALBI-T compared favorably with not only JIS but also $\mathrm{m}$-JIS in the ICG group. In addition, several patients in the present cohort were excluded due to a lack of data for Child-Pugh class (e.g., ascites, hepatic coma) or liver damage grade (ICG-r15). Thus, the simply calculated ALBI grade is thought to be useful for retrospective analysis as well as for predicting prognosis, especially for patients scheduled to undergo curative treatment such as resection or ablative therapy.

In Japan, HCC is often diagnosed at an earlier stage than in Western countries. Kudo et al. [31] recently reported that the number of cases of small-sized HCC has increased because recognition of the importance of surveillance for high-risk patients with chronic liver diseases has varied widely in Japan. Nevertheless, the prognosis for Japanese patients with HCC has improved [31]. Minagawa et al. [6] reported that LCSGJ TNM staging was more appropriate for early-stage HCC cases, while JIS has been proposed as a better total staging system than CLIP scoring $[5,9,32]$. On the other hand, the recent trend for HCC etiology in Japan has rapidly changed $[33,34]$. The main etiology of HCC in Japanese patients has been reported to be HCV infection $[33,35]$. Development of direct-acting antiviral drug therapy for HCV [36, 37] has led to an extremely high rate of HCV elimination, allowing for maintenance of hepatic function without progression of hepatic fibrosis after sustained virologic response (SVR). However, treatment of HCC after SVR with direct-acting antiviral drug therapy remains controversial, because the incidence of HCC after SVR in a group of interferon-free patients was reported to be $>2$-fold higher than that in an interferon-based therapy group (7.29 vs. $3.09 \%$ and 6.23 vs. 3.01\%) [38]. In addition, the number of nonBnonC HCC cases has been increasing $[33,34]$ in association with the aging of society. 
Based on these dynamic changes, it is expected that the frequency of HCC among Japanese patients with better hepatic function will increase. In addition, because of the progress in techniques used for imaging, and therapeutic assistant modalities for the diagnosis and treatment of HCC (virtual ultrasonography [39], artificial effusion [40], contrast-enhanced ultrasonography [41, 42], and Gd-EOB-DTPA-enhanced MRI [43, 44]), the frequencies of resection and ablative therapies including radiofrequency ablation will increase; thus, a more suitable hepatic function assessment tool for the recent trend in Japanese HCC is needed. In addition to the usefulness of ALBI-T for Japanese patients, that of modified CLIP scoring [45], modified BCLC (Barcelona Clinic Liver Cancer) staging [46], and BCLC-B substaging using ALBI grading instead of the Child-Pugh classification/scoring system [47] has recently been reported. Thus, the simply calculated ALBI grade can play an important role in the assessment of hepatic function not only in the recent trend of Japanese HCC but also worldwide.

A previous report noted that liver damage grading is now recognized to be superior as a hepatic functional assessment tool, and that the prognosis prediction value of $\mathrm{m}$-JIS is better than that of JIS [10]. Although the prognostic stratification ability (MST of each score) of m-JIS is thought to be superior to that of JIS, the AIC value was similar to that of JIS and ALBI-T. Liver damage grading is used for the assessment of hepatic function in the Evidence-Based Clinical Practice Guidelines for HCC of the JSH [2, 3], though data for the ICG-r15 are frequently lacking because ICG injection is required. In fact, the ICG-r15 was examined in only $66.4 \%$ of the patients of the total group. ALBI-T may have a noninferior predictive value, not only as compared to that of JIS but also to that of m-JIS even in patients who undergo ICG testing, while ALBI-T might show a better MST value than JIS and m-JIS. Moreover, the advantages of the ALBI grade are that it is calculated using only 2 common factors (albumin and total bilirubin) and determined based on serial ALBI scores as compared with other methods. In our previous study, there was a good correlation between ICG-r15 rate and ALBI score $(r=0.616 ; p<0.001)$, and the cutoff value for ALBI score for the ICG-r15 $(<30 \%$ ) was -2.222 (AUC 0.843 ; sensitivity 0.823 ; specificity 0.708 ; 95\% CI: 0.827-0.859) [48]. In the present analysis, a similar correlation between ALBI score and ICG-r15 rate was noted, as well as for the cutoff value for ALBI score (-2.270; AUC 0.828) for the ICG-r15 $(<30 \%)$, which is a cutoff indicator for subsegmental cases in the Makuuchi criteria $[22,23]$. Because subsegmentectomy has been thought to be the minimal anatomical surgical resection for HCC, we used the ICG-r15 $(<30 \%)$ for the cutoff indicator of subgrading for ALBI grade 2. Thus, we consider that a division into 4 ALBI grades can be conducted by making 2 subgrades for ALBI grade 2 ( $2 \mathrm{a}$ and $2 \mathrm{~b}$ ) based on the cutoff value (ALBI score -2.270 ) (mALBI grade). For each TNM stage, a good prognostic stratification ability according to mALBI grade was observed. As a result, mALBI-T showed a more detailed stratification among all of our HCC patients, as well as the best value for the AIC among the 4 tested scoring systems for early-stage HCC (Milan criteria) among patients treated curatively. mALBI and mALBI-T might provide more important information for decision-making regarding therapy.

In conclusion, we evaluated the prognostic significance of ALBI grading/ALBI-T for patients with HCC in Japan using data from a nationwide follow-up survey, and confirmed that they were applicable as well as the Child-Pugh classification/JIS and liver damage grading/ $\mathrm{m}$-JIS. Moreover, ALBI scoring might have potential for a division into additional grades for a more detailed assessment of hepatic function and prognosis, such as the present proposed mALBI grading and mALBI-T. Prospective examinations are needed for confirmation.

\section{Acknowledgement}

The authors express their gratefulness to Dr. Natsumi Yamashita, Shikoku Cancer Center, for the excellent advice. 
Hiraoka et al.: Validation and Potential of ALBI Grade and Prognostication in a Nationwide Survey of 46,681 HCC Patients in Japan

\section{Disclosure Statement}

The authors have no conflicts of interest to declare in regard to this study.

\section{References}

1 Pugh RN, Murray-Lyon IM, Dawson JL, Pietroni MC, Williams R: Transection of the oesophagus for bleeding oesophageal varices. Br J Surg 1973;60:646-649.

2 Makuuchi M, Kokudo N, Arii S, Futagawa S, Kaneko S, Kawasaki S, Matsuyama Y, et al: Development of evidencebased clinical guidelines for the diagnosis and treatment of hepatocellular carcinoma in Japan. Hepatol Res 2008;38:37-51.

3 Japan Society of Hepatology: Clinical Practice Guidelines for Hepatocellular Carcinoma 2013. http://www.jsh. or.jp/English/guidelines_en/Guidelines_for_hepatocellular_carcinoma_2013.

4 Johnson PJ, Berhane S, Kagebayashi C, Satomura S, Teng M, Reeves HL, O’Beirne J, et al: Assessment of liver function in patients with hepatocellular carcinoma: a new evidence-based approach - the ALBI grade. J Clin Oncol 2015;33:550-558.

5 Kudo M, Chung H, Osaki Y: Prognostic staging system for hepatocellular carcinoma (CLIP score): its value and limitations, and a proposal for a new staging system, the Japan Integrated Staging Score (JIS score). J Gastroenterol 2003;38:207-215.

6 Minagawa M, Ikai I, Matsuyama Y, Yamaoka Y, Makuuchi M: Staging of hepatocellular carcinoma: assessment of the Japanese TNM and AJCC/UICC TNM systems in a cohort of 13,772 patients in Japan. Ann Surg 2007;245: 909-922.

7 Liver Cancer Study Group of Japan: The General Rules for the Clinical and Pathological Study of Primary Liver Cancer, ed 6. Tokyo, Kanehara, 2015.

8 Prospective validation of the CLIP score: a new prognostic system for patients with cirrhosis and hepatocellular carcinoma. The Cancer of the Liver Italian Program (CLIP) Investigators. Hepatology 2000;31:840-845.

9 Kudo M, Chung H, Haji S, Osaki Y, Oka H, Seki T, Kasugai H, et al: Validation of a new prognostic staging system for hepatocellular carcinoma: the JIS score compared with the CLIP score. Hepatology 2004;40:1396-1405.

10 Nanashima A, Sumida Y, Abo T, Shindou H, Fukuoka H, Takeshita H, Hidaka S, et al: Modified Japan Integrated Staging is currently the best available staging system for hepatocellular carcinoma patients who have undergone hepatectomy. J Gastroenterol 2006;41:250-256.

11 Kudo M: Albumin-bilirubin grade and hepatocellular carcinoma treatment algorithm. Liver Cancer 2017;6: 185-188.

12 Hiraoka A, Kumada T, Michitaka K, Toyoda H, Tada T, Ueki H, Kaneto M, et al: Usefulness of albumin-bilirubin grade for evaluation of prognosis of 2,584 Japanese patients with hepatocellular carcinoma. J Gastroenterol Hepatol 2016;31:1031-1036.

13 Mazzaferro V, Regalia E, Doci R, Andreola S, Pulvirenti A, Bozzetti F, Montalto F, et al: Liver transplantation for the treatment of small hepatocellular carcinomas in patients with cirrhosis. N Engl J Med 1996;334:693-699.

14 Shiina S, Niwa Y, Shiratori Y, Terano A, Omata M: Percutaneous ethanol injection therapy for hepatocellularcarcinoma [sic!] (review). Int J Oncol 1993;2:669-675.

15 Shiina S, Teratani T, Obi S, Hamamura K, Koike Y, Omata M: Percutaneous ethanol injection therapy for liver tumors. Eur J Ultrasound 2001;13:95-106.

16 Tateishi R, Shiina S, Teratani T, Obi S, Sato S, Koike Y, Fujishima T, et al: Percutaneous radiofrequency ablation for hepatocellular carcinoma. An analysis of 1,000 cases. Cancer 2005;103:1201-1209.

17 Hiraoka A, Michitaka K, Horiike N, Hidaka S, Uehara T, Ichikawa S, Hasebe A, et al: Radiofrequency ablation therapy for hepatocellular carcinoma in elderly patients. J Gastroenterol Hepatol 2010;25:403-407.

18 Shiina S, Tateishi R, Arano T, Uchino K, Enooku K, Nakagawa H, Asaoka Y, et al: Radiofrequency ablation for hepatocellular carcinoma: 10-year outcome and prognostic factors. Am J Gastroenterol 2012;107:569-577; quiz 578.

19 Akaike H: Information theory and an extension of the maximum likelihood principle; in Petrov BN, Csaki F (eds): Proceedings of the 2nd International Symposium on Information Theory. Budapest, Akademiai Kiado, 1973, pp 267-281.

20 Kanda Y: Investigation of the freely available easy-to-use software "EZR" for medical statistics. Bone Marrow Transplant 2013;48:452-458.

21 Takayasu K, Arii S, Ikai I, Omata M, Okita K, Ichida T, Matsuyama Y, et al: Prospective cohort study of transarterial chemoembolization for unresectable hepatocellular carcinoma in 8,510 patients. Gastroenterology 2006;131:461-469.

22 Miyagawa S, Makuuchi M, Kawasaki S, Kakazu T: Criteria for safe hepatic resection. Am J Surg 1995;169:589594.

23 Seyama Y, Kokudo N: Assessment of liver function for safe hepatic resection. Hepatol Res 2009;39:107-116.

24 Okuda K, Ohtsuki T, Obata H, Tomimatsu M, Okazaki N, Hasegawa H, Nakajima Y, et al: Natural history of hepatocellular carcinoma and prognosis in relation to treatment. Study of 850 patients. Cancer 1985;56:918-928. 
Hiraoka et al.: Validation and Potential of ALBI Grade and Prognostication in a Nationwide Survey of 46,681 HCC Patients in Japan

25 Tateishi R, Yoshida H, Shiina S, Imamura H, Hasegawa K, Teratani T, Obi S, et al: Proposal of a new prognostic model for hepatocellular carcinoma: an analysis of 403 patients. Gut 2005;54:419-425.

26 Toyoda H, Kumada T, Osaki Y, Oka H, Urano F, Kudo M, Matsunaga T: Staging hepatocellular carcinoma by a novel scoring system (BALAD score) based on serum markers. Clin Gastroenterol Hepatol 2006;4:1528-1536.

27 Berhane S, Toyoda H, Tada T, Kumada T, Kagebayashi C, Satomura S, Schweitzer N, et al: Role of the GALAD and BALAD-2 serologic models in diagnosis of hepatocellular carcinoma and prediction of survival in patients. Clin Gastroenterol Hepatol 2016;14:875-886.e6.

28 Toyoda H, Tada T, Johnson PJ, Izumi N, Kadoya M, Kaneko S, Kokudo N, et al: Validation of serological models for staging and prognostication of HCC in patients from a Japanese nationwide survey. J Gastroenterol 2017, Epub ahead of print.

29 Chan AW, Chong CC, Mo FK, Wong J, Yeo W, Johnson PJ, Yu S, et al: Applicability of albumin-bilirubin-based Japan Integrated Staging score in hepatitis B-associated hepatocellular carcinoma. J Gastroenterol Hepatol 2016;31:1766-1772.

30 Harimoto N, Yoshizumi T, Sakata K, Nagatsu A, Motomura T, Itoh S, Harada N, et al: Prognostic significance of combined albumin-bilirubin and tumor-node-metastasis staging system in patients who underwent hepatic resection for hepatocellular carcinoma. Hepatol Res 2017, Epub ahead of print.

31 Kudo M, Izumi N, Sakamoto M, Matsuyama Y, Ichida T, Nakashima O, Matsui O, et al: Survival analysis over 28 years of 173,378 patients with hepatocellular carcinoma in Japan. Liver Cancer 2016;5:190-197.

32 Kudo M: Real practice of hepatocellular carcinoma in Japan: conclusions of the Japan Society of Hepatology 2009 Kobe Congress. Oncology 2010;78(suppl 1):180-188.

33 Tateishi R, Okanoue T, Fujiwara N, Okita K, Kiyosawa K, Omata M, Kumada H, et al: Clinical characteristics, treatment, and prognosis of non-B, non-C hepatocellular carcinoma: a large retrospective multicenter cohort study. J Gastroenterol 2015;50:350-360.

34 Hiraoka A, Ochi M, Matsuda R, Aibiki T, Okudaira T, Kawamura T, Yamago H, et al: Ultrasonography screening for hepatocellular carcinoma in Japanese patients with diabetes mellitus. J Diabetes 2016;8:640-646.

35 Ikai I, Arii S, Okazaki M, Okita K, Omata M, Kojiro M, Takayasu K, et al: Report of the 17th Nationwide FollowUp Survey of Primary Liver Cancer in Japan. Hepatol Res 2007;37:676-691.

36 Su F, Beste LA, Green PK, Berry K, Ioannou GN: Direct-acting antivirals are effective for chronic hepatitis C treatment in elderly patients: a real-world study of 17,487 patients. Eur J Gastroenterol Hepatol 2017;29: 686-693.

37 Toyoda H, Kumada T, Tada T, Shimada N, Takaguchi K, Senoh T, Tsuji K, et al: Efficacy and tolerability of an IFN-free regimen with DCV/ASV for elderly patients infected with HCV genotype 1B. J Hepatol 2017;66:521527.

38 Toyoda H, Tada T, Takaguchi K, Senoh T, Shimada N, Hiraoka A, Michitaka K, et al: Differences in background characteristics of patients with chronic hepatitis $\mathrm{C}$ who achieved sustained virologic response with interferonfree versus interferon-based therapy and the risk of developing hepatocellular carcinoma after eradication of hepatitis C virus in Japan. J Viral Hepat 2017;24:472-476.

39 Hirooka M, Iuchi H, Kumagi T, Shigematsu S, Hiraoka A, Uehara T, Kurose K, et al: Virtual sonographic radiofrequency ablation of hepatocellular carcinoma visualized on CT but not on conventional sonography. AJR Am J Roentgenol 2006;186:S255-S260.

40 Uehara T, Hirooka M, Ishida K, Hiraoka A, Kumagi T, Kisaka Y, Hiasa Y, et al: Percutaneous ultrasound-guided radiofrequency ablation of hepatocellular carcinoma with artificially induced pleural effusion and ascites. J Gastroenterol 2007;42:306-311.

41 Hiraoka A, Ichiryu M, Tazuya N, Ochi H, Tanabe A, Nakahara H, Hidaka S, et al: Clinical translation in the treatment of hepatocellular carcinoma following the introduction of contrast-enhanced ultrasonography with Sonazoid. Oncol Lett 2010;1:57-61.

42 Hiraoka A, Hiasa Y, Onji M, Michitaka K: New contrast enhanced ultrasonography agent: impact of Sonazoid on radiofrequency ablation. J Gastroenterol Hepatol 2011;26:616-618.

43 Di Martino M, Marin D, Guerrisi A, Baski M, Galati F, Rossi M, Brozzetti S, et al: Intraindividual comparison of gadoxetate disodium-enhanced MR imaging and 64-section multidetector CT in the detection of hepatocellular carcinoma in patients with cirrhosis. Radiology 2010;256:806-816.

44 Sano K, Ichikawa T, Motosugi U, Sou H, Muhi AM, Matsuda M, Nakano M, et al: Imaging study of early hepatocellular carcinoma: usefulness of gadoxetic acid-enhanced MR imaging. Radiology 2011;261:834-844.

45 Chan AW, Chong CC, Mo FK, Wong J, Yeo W, Johnson PJ, Yu S, et al: Incorporating albumin-bilirubin grade into the Cancer of the Liver Italian Program system for hepatocellular carcinoma. J Gastroenterol Hepatol 2017; $32: 221-228$.

46 Chan AW, Kumada T, Toyoda H, Tada T, Chong CC, Mo FK, Yeo W, et al: Integration of albumin-bilirubin (ALBI) score into Barcelona Clinic Liver Cancer (BCLC) system for hepatocellular carcinoma. J Gastroenterol Hepatol 2016;31:1300-1306.

47 Hiraoka A, Kumada T, Nouso K, Tsuji K, Itobayashi E, Hirooka M, Kariyama K, et al: Proposed new sub-grouping for intermediate-stage hepatocellular carcinoma using albumin-bilirubin grade. Oncology 2016;91:153-161.

48 Hiraoka A, Kumada T, Kudo M, Hirooka M, Tsuji K, Itobayashi E, Kariyama K, et al: Albumin-bilirubin (ALBI) grade as part of the evidence-based clinical practice guideline for HCC of the Japan Society of Hepatology: a comparison with the liver damage and Child-Pugh classifications. Liver Cancer 2017;6:204-215. 